Documentation et bibliothèques

DOCUMENTATION BIBLIOTHËQUES

\title{
Le Centre de documentation du Grand conseil des Cris du Québec
}

\section{André Boulanger}

Volume 33, numéro 4, octobre-décembre 1987

URI : https://id.erudit.org/iderudit/1052556ar

DOI : https://doi.org/10.7202/1052556ar

Aller au sommaire du numéro

\section{Éditeur(s)}

Association pour l'avancement des sciences et des techniques de la documentation (ASTED)

\section{ISSN}

0315-2340 (imprimé)

2291-8949 (numérique)

Découvrir la revue

\section{Citer cet article}

Boulanger, A. (1987). Le Centre de documentation du Grand conseil des Cris du Québec. Documentation et bibliothèques, 33(4), 127-129.

https://doi.org/10.7202/1052556ar

Tous droits réservés (C) Association pour l'avancement des sciences et des techniques de la documentation (ASTED), 1987
Ce document est protégé par la loi sur le droit d'auteur. L'utilisation des services d'Érudit (y compris la reproduction) est assujettie à sa politique d'utilisation que vous pouvez consulter en ligne.

https://apropos.erudit.org/fr/usagers/politique-dutilisation/ 


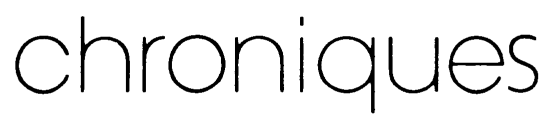

\section{Le Centre de documentation du Grand conseil des Cris du Québec}

Les neuf mille Cris du Québec habitent entre le $49 \mathrm{e}$ et le $56 \mathrm{e}$ parallèle. Ils sont répartis en neuf communautés: Whapmagoostui, Chisasibi, Wemindji, Eastmain, Waskaganish, Nemaska, Mistassini, Waswanipi et Ouje-bougamau. Cette dernière communauté a été nouvellement crée pour les Cris de Chibougamau afin qu'ils puissent obtenir des gouvernements fédéral et provincial des terres où résider et y préserver leur culture.

L'événement important des deux dernières décennies pour la reconnaissance de leurs droits a été la Convention de la Baie James et du Nord québécois. La convention a permis de créer un certain nombre d'organismes cris dont l'objectif est d'assurer le développement économique et social. Parmi les plus importants on retrouve le Grand conseil des Cris (du Québec), I'Administration régionale crie, la Commission scolaire crie et le Conseil cri de la santé et des services sociaux.

Les deux principaux organismes de niveau politique et économique sont le Grand conseil des Cris (du Québec) et l'Administration régionale crie. Le premier, formé en 1974, est le représentant politique des Cris. Son principal objectif est d'assister et de défendre le peuple cri du Québec. II fut chargé de négocier les ententes antérieures à la signature de la Convention de la Baie James et du Nord québécois. II s'intéresse à d'autres grands dossiers comme l'application de la Convention ou la défense des intérêts des autochtones au niveau national et international.

Le second organisme, l'Administration régionale crie, fut créé par une loi québécoise. Son principal objectif est de promouvoir le développement économique et social, de défendre les intérêts cris à l'intérieur des divers organismes consultatifs ou décisionnels créés par la Convention et à offrir des services aux différentes communautés cries.

\section{Le centre de documentation}

Le centre de documentation a été créé en 1981. II doit conserver et rendre accessible toute l'information accumulée concernant les Cris et le territoire de la Baie James. Andrew Preston a mis sur pied le centre de documentation. $\mathrm{Ce}$ centre comporte trois sections: une bibliothèque, une cartothèque et des archives.

La bibliothèque contient plus de 4000 titres. Les principaux sujets couverts sont les nombreux aspects de la vie des Cris, les différents projets hydro-électriques de la Baie James, l'art autochtone, les publications du ministère des Affaires indiennes, etc.

Pour sa part, la cartothèque est constituée d'une collection de cartes géographiques et topographiques, de plans, des index de photographies aériennes de la région de la Baie James.

La section archives, troisième section du centre, est la plus importante en volume. Elle comprend plus de 375 boîtes d'archives qui regroupent les documents inactifs et de valeur historique du Grand conseil, de l'Administration régionale et d'autres organismes cris. Ces documents renferment surtout de la correspondance, des procèsverbaux de réunions, des études et de la documentation éphémère.

Les principaux usagers du centre sont le personnel du Grand conseil et de l'Administration régionale crie ainsi que les chercheurs intéressés par la nation crie et sa culture. La pratique du prêt est limitée étant donnée la nature confidentielle de plusieurs documents. Actuellement, il est difficile de mesurer l'ampleur de la collection puisqu'il n'existe pas de catalogue imprimé. II devrait y en avoir un cependant d'ici deux ou trois mois pour la section bibliothèque.

\section{Le problème de classification}

Pour illustrer ce problème, prenons un exemple: il existait un catalogue "Auteur-Titre" manuscrit pour la bibliothèque. C'était un inventaire provisoire. II était difficile d'y retrouver l'information; les différents rayons étaient identifiés au nom des organismes comme dans la section des archives et il n'y avait pas d'ordre de classement alphabétique mais un code numérique tout simplement. 
Pour résoudre ce problème, on a ordonné tous les volumes par ordre alphabétique de titres. On a alors commencé à classer les livres à l'aide d'un micro-ordinateur, en essayant de respecter les normes de classement en usage ainsi que celles de la deuxième édition des Règles de catalogage anglo-américaines (AACR 2). De plus, le logiciel utilisé, Microsoft File, avait aussi ses exigences.

On peut considérer l'ensemble des notices comme une base de données plutôt qu'un fichier de bibliothèque. Le centre utilise les éléments suivants: auteur, titre, autres auteurs, sous-titre, édition, cote et vedettes-matière.

Il a fallu adapter certaines règles aux besoins locaux et au logiciel en usage. Par exemple, l'entrée principale est consacrée à l'auteur mais, s'il y en a plus de trois, elle l'est au titre et on indique le premier auteur comme deuxième auteur; de même, on a omis tous les articles en début de titre parce qu'ils pouvaient occasionner des erreurs; on utilise les vedettes-matière en anglais peu importe la langue de publication des ouvrages; enfin, pour une publication en série, le titre de la série apparaît dans la zone du soustitre.

\section{Les vedettes-matière}

Le problème des vedettes-matière est lié au fait que les répertoires ne donnent pas beaucoup de sujets concernant les autochtones du Canada et du Québec. Le SEARS, par exemple, contient 40 vedettes sur les autochtones; le Canadian Subject Headings en a seulement 85 . En somme, ces éléments ne représentent que peu d'aspects de la vie des autochtones.

II faut donc établir des sujets maison en respectant une certaine logique. L'adoption d'un vocabulaire libre est à proscrire, car il demande beaucoup de contrôles et de vérifications. Les sujets maison sont établis à partir du SEARS et du Canadian Subject Headings auxquels on ajoute la mention "Cree Indians-Quebec" ou "Native Peoples-Quebec». Cette façon de faire permet de bien clarifier le sujet et de garder un ordre logique.

Si l'on veut trouver, dans une bibliothèque ordinaire, tous les volumes qui portent sur l'enseignement secondaire des indiens du Québec ou sur des compagnies aériennes cries, il ne sera pas possible de les identifier directement puisqu'ils vont être signalés sous la vedette de base. Chez nous, ils seront à deux sujets: «Native Peoples-Quebec-Education, Secondary» et "Cree Indians-Quebec-Aeronautics, Commercial».

Le problème des petits centres de documentation est de trouver des outils adéquats. Actuellement le SEARS est le meilleur outil, semble-t-il: il ne coûte pas cher et il est très flexible. Malheureusement, il n'existe pas d'équivalent en français. Dans un proche avenir, il faudra publier un répertoire qui reflètera les aspirations des peuples autochtones d'Amérique.

\section{La cote}

Plusieurs bibliothèques ont une cote ou un code facilitant le repérage des volumes sur les rayons. II existe divers ordres de classement (code de couleurs, auteur, titre, types d'usagers: enfants, adultes, etc.). Dans la bibliothèque du centre, il était impossible d'utiliser l'auteur et le titre, car plusieurs livres ont le même auteur: le Grand conseil des Cris (du Québec) et l'Administration régionale crie ou le même titre (brief, proposal, report, research).

II est possible d'utiliser divers systèmes pour établir la cote. Nous avons éliminé le schéma de classement de la Library of Congress (LC), car il était trop volumineux ( 30 volumes) et trop coûteux et il est surtout recommandé pour des bibliothèques importantes. Nous avons décidé d'utiliser le Dewey $(D D C)$ parce qu'il répondait mieux aux besoins du centre et qu'il reflétait les sujets propres aux autochtones du Québec et du Canada.

En bref, la cote Dewey est facile à comprendre pour les usagers, on peut l'adapter à des besoins spécifiques, on a le choix entre une édition abrégée et une édition intégrale (3 volumes). De plus, si l'on fait du catalogage original, son utilisation est plus facile que celle du schéma de la Library of Congress et la dispersion des titres concernant les Cris, les autochtones du Québec et du Canada, est possible à l'intérieur des schémas de classification (ce qui ne l'est pas avec le LC).

Voyons deux exemples:

a) $\mathbf{3 3 0 . 0 8 9 9 7}=$ Cree Indians-Quebec-Economic conditions

$330=$ =Économie

$330.089=$ Traitement selon l'origine ethnique, raciale, nationale

$330.08997=$ Traitement pour les Cris et les autres nations autochtones

b) $970.57141=$ =ree Indians-QuebecGovernment relations

$970.5=$ Native Peoples-Government relations

$970.571=$ Native Peoples-CanadaGovernment relations

970.5714 =Native Peoples-QuebecGovernment relations

$\mathbf{9 7 0 . 5 7 1 4 1}=$ =Cree Indians-QuebecGovernment relations (les Cris vivent dans le nord du Québec) 
Le complément de l'indice Dewey est le chiffre d'auteur établi à l'aide des tables Cutter-Sanborn. On aura ainsi A567c pour Andrews, Helen plus la première lettre du titre.

\section{Le catalogage maison: les vedettes-matière et la cote}

Pour résoudre certains problèmes, nous avons dû ajouter de nouvelles vedettes: noms des actuels ou futurs projets hydro-électriques de la région de la Baie James (La Grande, NBR, Grande Baleine, Eastman-Opinaca), noms des diverses communautés cries. Nous avons appliqué la même solution pour la cote en y ajoutant l'année de publication. Voici quelques exemples:

a) Établissement hydro-électrique NottawayBroadback-Rupert (NBR)

$\begin{array}{ll}970.47141 & =\text { cote Dewey } \\ \text { NBR } & =\text { code du «projet» Nottaway- } \\ & \text { Broadback-Rupert } \\ \text { A729b } & =\text { chiffre d'auteur } \\ 1976 & =\text { année de publication }\end{array}$

b) Communauté crie de Nemaska

$\begin{array}{ll}970.47141 & =\text { cote Dewey } \\ \text { NEM } & =\text { code de la communauté crie de } \\ & \text { Nemaska } \\ \text { A666c } & =\text { chiffre d'auteur } \\ 1984 & =\text { année de publication }\end{array}$

Toutes ces adaptations ont été faites, en somme, pour faciliter la recherche assistée par ordinateur et pour éviter les embouteillages sous certaines cotes.

\section{Conclusion}

La section bibliothèque est une partie importante du centre de documentation avec celles des archives et de la carthothèque. Elle représente une bonne source d'information sur les Cris du Québec. Notre objectif le plus important est de rendre la documentation accessible aux usagers. Actuellement, nous établissons un catalogue de la collection de la bibliothèque et d'une bonne partie des archives grâce au logiciel Microsoft File. Sans l'informatique, nous ne pourrions retrouver un document facilement et dans un temps relativement court.

\section{André Boulanger}

Centre de documentation

Grand conseil des Cris

Val-d'or (Québec) 